\title{
Role of nitric oxide synthase in the development of bone cancer pain and effect of L-NMMA
}

\author{
YAN YANG ${ }^{*}$, JUAN ZHANG $^{*}$, YUE LIU, YAGUO ZHENG, JINHUA BO, \\ XIAOFANG ZHOU, JUNHUA WANG and ZHENGLIANG MA \\ Department of Anesthesiology, Affiliated Drum-Tower Hospital, \\ Medical School of Nanjing University, Nanjing, Jiangsu 210008, P.R. China
}

Received December 28, 2014; Accepted November 10, 2015

DOI: $10.3892 / \mathrm{mmr} .2015 .4647$

\begin{abstract}
Spinal nitric oxide is involved in the mechanisms of pain generation and transmission during inflammatory and neuropathic pain. The aim of the present study was to explore the role of spinal nitric oxide in the development of bone cancer pain. $2 \times 10^{5}$ osteosarcoma cells were implanted into the intramedullary space of right femurs of $\mathrm{C} 3 \mathrm{H} / \mathrm{HeJ}$ mice to induce a model of ongoing bone cancer. Polymerase chain reaction and immunohistochemical analyses were performed to assess the expression of neuronal nitric oxide synthase (nNOS) and inducible (i)NOS in the spinal cord following inoculation. The results showed that inoculation of osteosarcoma cells induced progressive bone cancer, accompanied with pain-associated behavior. The levels of nNOS mRNA in the spinal cord of tumor mice began to increase at day 10 and then decreased to the level in sham mice at day 14, while iNOS mRNA markedly increased in the tumor group at days 10 and 14. Immunohistochemical analysis showed that nNOS- and iNOS-positive neurons were mainly located in the superficial dorsal horn and around the central canal of the L3-L5 spinal cord. Intrathecal injection of $50 \mu \mathrm{g}$ NOS inhibitor $\mathrm{N}^{\mathrm{G}}$-monomethyl-L-arginine (L-NMMA) attenuated cancer-evoked pain behaviors at day 14. These findings indicated that an upregulation of nNOS and iNOS in the spinal cord is associated with bone cancer pain and suggests that exogenously administered L-NMMA may have beneficial effects to alleviate bone cancer pain.
\end{abstract}

Correspondence to: Professor Zhengliang Ma, Department of Anesthesiology, Affiliated Drum-Tower Hospital, Medical School of Nanjing University, 321 Zhongshan Road, Nanjing, Jiangsu 210008, P.R. China

E-mail: mazhengliang1964@nju.edu.cn

*Contributed equally

Key words: nitric oxide synthase, bone cancer pain, L-NMMA

\section{Introduction}

Bone cancer pain is a type of severe and chronic pain that affects the quality of life in cancer patients; however, no breakthrough regarding the elucidation of underlying mechanisms and the development of therapeutics for bone cancer pain has been achieved (1). Studies have indicated that nitric oxide (NO) is an important neurotransmitter contributing to the development and maintenance of central and peripheral sensitization in inflammatory (2) and neuropathic pain (3).

NO is synthesized from L-arginine via a family of nitric oxide synthases (NOS), which are key enzymes in NO biosynthesis and comprise neuronal NOS (nNOS), endothelial NOS (eNOS) and inducible NOS (iNOS). The pathophysiological functions of $\mathrm{NO}$ are regulated by the expression and activity of these isoforms $(4,5)$. nNOS, expressed in the nervous system, produces NO in neurons and has been shown to contribute to spinal nociceptive processing in several pain models (6-12). iNOS, which is not constitutively expressed and must be induced to be synthesized, is abundant in a variety of cell types, including glial cells, macrophages, chondrocytes and neutrophils (13). Previous studies demonstrated that iNOS is also involved in the mechanisms of inflammatory, neuropathic pain $(5,14)$ as well as herpetic allodynia $(15)$.

Bone cancer pain is thought to have inflammatory, neuropathic and tumorigenic components. A previous study by our group has shown that NR2B-containing $N$-methyl-D-aspartate (NMDA) receptor has a critical role in spinal nociceptive processing during bone cancer pain (16). Activation of NMDA receptors allows $\mathrm{Ca}^{2+}$ to enter neurons. Increased intracellular $\mathrm{Ca}^{2+}$ triggers a cascade of events that includes activation of nNOS, leading to the production of NO in the spinal cord (17). Another previous study by our group also demonstrated that upregulation of NR2B and nNOS expression in the spinal dorsal horn contributes to hyperalgesia induced by chronic compression of dorsal root ganglia (18). However, the role of NOS in the development of bone cancer pain has remained elusive.

The present study used a murine model of osteosarcoma-associated bone cancer pain, to explore whether NOS in the spinal L3-L5 segments is responsible for the development of bone cancer pain and to determine the efficacy of $\mathrm{N}^{\mathrm{G}}$-monomethyl-L-arginine (L-NMMA) administration in alleviating bone cancer pain. 


\section{Materials and methods}

Animals. Experiments were approved by the Animal Care and Use Committee of the Medical School of Nanjing University (Nanjing, China) and were in accordance with the guidelines for the use of laboratory animals (19). The numbers of animals and their suffering were minimized in all cases. Male $\mathrm{C} 3 \mathrm{H} / \mathrm{HeJ}$ mice ( $\mathrm{n}=100$; weight, 20-22 g; 4-6 weeks old), purchased from the Model Animal Research Center of Nanjing University (Nanjing, China), were housed in a temperature-controlled $\left(21 \pm 1^{\circ} \mathrm{C}\right)$ room with a 12 -h light/dark cycle with access to food and water ad libitum. Each group used to analyze behavior contained 8 mice, and each group in PCR or immunohistochemistry study contained 5 mice. The mice subjected to the behavioral studies were not processed for PCR/immunohistochemistry, and were euthanized after the last behavioral test.

Cell culture and tumor-cell inoculation. The NCTC 2472 osteosarcoma cell line (no. 2087787; American Type Culture Collection, Manassas, VA, USA) was used in the present study. The cells were incubated in NCTC 135 medium (Sigma-Aldrich, St. Louis, USA) containing 10\% horse serum (Gibco; Thermo Fisher Scientific, Inc., Waltham, MA, USA) in a humidified atmosphere containing $5 \% \mathrm{CO}_{2}$ at $37^{\circ} \mathrm{C}$.

The mouse model of bone cancer was established according to the method of previous studies by our and another group (16,20). Briefly, $1 \%$ pentobarbital sodium $(50 \mathrm{mg} / \mathrm{kg}$; Sigma-Aldrich) in normal saline [Baxter Healthcare (Tianjin) Co. Ltd., Tianjin, China] was administered to the mice by intraperitoneal injection and the right femur condyle was perforated with a 30-gauge needle (Jiangsu Zhengkang Medical Equipment Co., Ltd., Changzhou, China). Subsequently, $2 \times 10^{5}$ NCTC 2472 cells in $20 \mu 1 \alpha$-minimum essential medium (Thermo Fisher Scientific, Inc.) were injected into the intramedullary space of the femur. In the sham group, the medium contained no cells. Subsequently, the injection hole was sealed with dental amalgam (AT\&M Biomaterials Co., Ltd., Beijing, China), followed by copious irrigation with normal saline and closing of the wound.

In the interaction studies, mice were intrathecally administered $50 \mu \mathrm{g}$ L-NMMA (Cayman Chemical Company, Ann Arbor, Michigan, USA) dissolved in artificial cerebrospinal fluid $(10 \mu \mathrm{g} / \mu \mathrm{l} ; 5 \mu \mathrm{l}$ per mouse; Harvard Apparatus, Holliston, MA, USA) at post-operative day 14 , at which pain-associated behavior was observed. Mice in the sham group received vehicle treatment. Intrathecal injections were performed free-hand between the L5 and L6 lumbar space in unanesthetized male mice according to the method of Hylden and Wilcox (21). Pain-associated behavior was assessed prior to, as well as at 2, 12 and $24 \mathrm{~h}$ after L-NMMA administration.

The mice assigned for behavioral tests were sacrificed after the last measurement. The mice were deeply anesthetized with pentobarbital $(50 \mathrm{mg} / \mathrm{kg}$, intraperitoneally) and then sacrificed by cervical dislocation. The mice assigned for Reverse-transcription quantitative polymerase chain reaction (RT-qPCR) were individually sacrificed at post-operative days 7, 10 or 14 , and the spinal cord segments were immediately removed. The mice assigned for immunohistochemical staining were also deeply anesthetized with pentobarbital individually at post-operative days 7,10 or 14 , and then transcardially perfused with saline followed by $4 \%$ paraformaldehyde (PFA; Beijing Dingguo Changsheng Biotechnology Co., Ltd., Beijing, China).

Assessment of pain-associated behavior. Withdrawal thresholds as well as latency to mechanical and thermal stimulation of mice were examined prior to surgery as well as at post-operative days 3,5, 7, 10 and 14. Mice were allowed to acclimatize for at least $30 \mathrm{~min}$ prior to each test.

Mechanical allodynia. The paw withdrawal mechanical threshold (PWMT) was assessed according to the method by Chaplan et al (22), which was also used in a previous study by our group (16). Briefly, a set of von Frey filaments $(0.16,0.4$, 0.6, 1.0, 1.4 and 2.0g; Stoelting, Wood Dale, IL, USA) was used to vertically push against the plantar surface of the right hind paw. Each mouse was tested five times per stimulus force. The lightest von Frey filament that evoked three or more brisk withdrawal or paw flinching reactions was regarded as the PWMT.

Thermal hyperalgesia. The paw withdrawal thermal latency (PWTL) was measured according to a method of a previous study by our group (23) using a radiant thermal stimulator (BME410A; Institute of Biological Medicine, Academy of Medical Science, Tianjin, China) that focused onto the plantar surface of the hind paw. The PWTL was defined as the latency of the mice to lift or lick their hind paw. Each mouse was tested five times with a 5-min interval. A cut-off time of $20 \mathrm{sec}$ was used to avoid tissue damage. The mean PWTL was obtained from the latter three stimuli.

$R T-q P C R$. Following the sacrifice of the mice, the L3-L5 lumbar spinal cord segments were immediately harvested, frozen in liquid nitrogen and stored at $-80^{\circ} \mathrm{C}$. The spinal tissues were homogenized prior to storage by grinding in liquid nitrogen with a small mortar. Total RNA was isolated and purified using TRIzol (Invitrogen; Thermo Fisher Scientific, Inc.) following the manufacturer's instructions. RT was performed using a Moloney murine leukemia virus reverse transcriptase kit (Promega Corp., Madison, WI, USA). The generated cDNA was then used as a template for PCR amplification with Taq DNA polymerase and ROX reference dye provided by the SYBR Premix Ex Taq kit (DRR041A; Takara, Dalian, China) using StepOnePlus RT-PCR system (Applied Biosystems; Thermo Fisher Scientific, Inc.). $\beta$-actin, nNOS and iNOS were amplified using specific oligonucleotide primers: forward, 5'-AGGAGCAAGGAGGCCATATT-3' and reverse, 5'-AAC ACACCAGCATCCTCCTC-3' for nNOS; forward, 5'-TGA TGTGCTGCCTCTGGTCT-3' and reverse, 5'-ACTTCCTCC AGGATGTTGTA-3' for iNOS; and forward, 5'-GAGACC TTCAACACCCCAGC-3' and reverse, 5'-CACAGAGTACTT GCGCTCAG-3' for $\beta$-actin (GenScript, Piscataway, NJ, USA). $\beta$-actin was used as internal standard. PCR amplification was performed using the following thermocycling conditions: $94^{\circ} \mathrm{C}$ for $5 \mathrm{~min}$, followed by 35 cycles of $94^{\circ} \mathrm{C}$ for $30 \mathrm{sec}, 66^{\circ} \mathrm{C}$ (or $62^{\circ} \mathrm{C}$ for iNOS and $\beta$-actin) for $30 \mathrm{sec}$ and $72^{\circ} \mathrm{C}$ for $30 \mathrm{sec}$, and a final elongation at $72^{\circ} \mathrm{C}$ for $10 \mathrm{~min}$. The relative expression was calculated using the $\Delta \Delta \mathrm{Cq}$ method (24) and optimized with a standard curve to confirm specificity. Each sample $(5 \mu \mathrm{l})$ was electrophoresed on 2\% agarose gel (Biowest, Nuaillé, France) with ethidium bromide (Biomatik Corporation, Cambridge, 


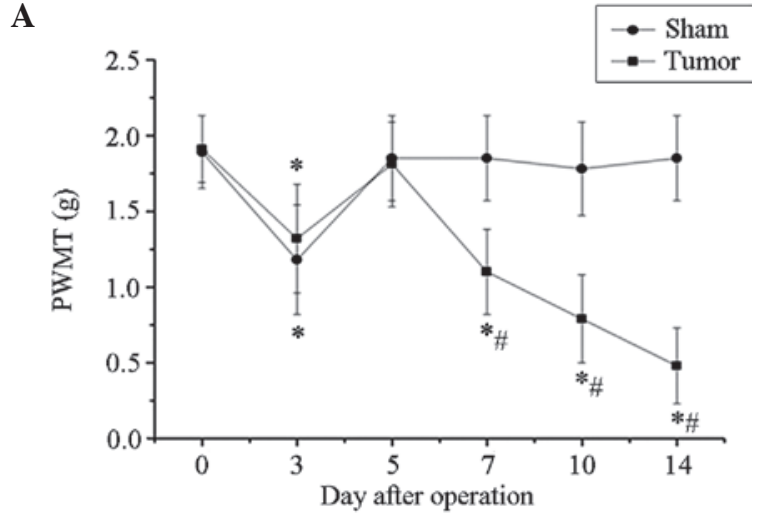

B

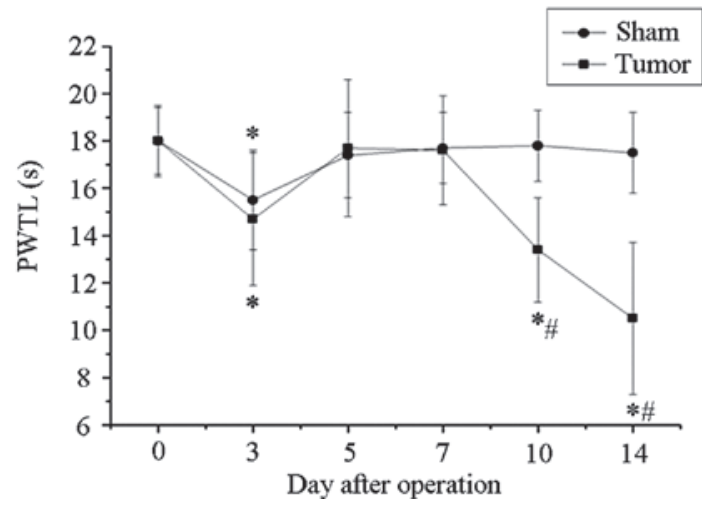

Figure 1. Tumor-bearing mice exhibited mechanical and thermal hyperalgesia (A) The righthind limb of mice in the tumor group displayed a significant decline of PWMT at post-operative day 7 and sustained to drop until day 14. (B) PWTL of mice in the tumor group showed a marked decrease at post-operative day 3 and recovered to basic levels at days 5 and 7. It decreased again at day 10 post-operation and reached the lowest level at day 14. Values are expressed as mean \pm standard deviation. ${ }^{*} \mathrm{P}<0.05$ vs. the same group of mice at day 0 , ${ }^{\text {"}} \mathrm{P}<0.05$ vs. sham group at the same time-point. PWTL, paw withdrawal thermal latency; PWTM, paw withdrawal mechanical threshold.

ON, Canada) and the intensity of each band was analyzed using a gel imaging analytical system (UVP GDS 8000; UVP, Upland, CA, USA). The sample without reverse transcriptase was used as a negative control and showed no detectable band.

Immunohistochemical staining. To localize and assess the expression of nNOS and iNOS in the spinal cord during bone cancer development, mice in the tumor and sham groups were transcardially perfused with saline, followed by freshly prepared $4 \%$ paraformaldehyde at post-operative days 7 , 10 and 14 (n=15 per group). The L3-L5 lumbar spinal cord segments were removed, fixed in $4 \%$ PFA overnight at $4{ }^{\circ} \mathrm{C}$ and then embedded in paraffin for sectioning ( $5 \mu \mathrm{m}$; five mice per group and ten sections from each mouse). The sections were dewaxed in xylene (Nanjing Chemical Reagent Co., Ltd., Nanjing, China) for 10 mins and hydrated with a graded ethanol series $(100,95,90,85,80$ and 70\%; Nanjing Chemical Reagent Co., Ltd.) for 3 mins each, at room temperature. The sections were washed in double distilled water (Nanjing Chemical Reagent Co., Ltd.) for 5 mins and boiled in $0.1 \mathrm{~mol} / 1$ sodium citrate buffer ( $\mathrm{pH}$ 6.0; Wuhan Boster Biological Technology, Ltd., Wuhan, China) for $20 \mathrm{~min}$ prior to exposure to $3 \%$ $\mathrm{H}_{2} \mathrm{O}_{2}$ (Shanghai Shenggong Biology Engineering Technology Service, Ltd., Shanghai, China) for $10 \mathrm{~min}$ to bleach endogenous peroxidases. Immunostaining was performed with rabbit primary polyclonal antibodies against nNOS (1:10,000; Sigma-Aldrich; SAB4502010) or rabbit primary polyclonal anti-iNOS (1:800; Sigma-Aldrich; SAB4502011) for $30 \mathrm{~min}$ at $37^{\circ} \mathrm{C}$, followed by washing with PBS 3 times for 10 mins. They were then incubated with a horseradish peroxidase-labeled goat anti-rabbit IgG (heavy and light chain; Beyotime Institute of Biotechnology; A0208) secondary antibody at $4^{\circ} \mathrm{C}$ overnight. The sections were then washed with PBS 3 times for 10 mins and visualized with diaminobenzidine (Beyotime Institute of Biotechnology, Haimen, China) and observed under a light microscope (Olympus DP11; Olympus, Tokyo, Japan). Optical density of the images was analyzed using Image-Pro Plus 6.0 analysis software (Media Cybernetics, Inc., Rockville, MD, USA). The mean optical density was obtained by averaging the values from five sections.

Statistical analysis. Values are expressed as the mean \pm standard deviation. Animals were assigned to various treatment groups in a randomized way. Repeated-measures analysis of variance (ANOVA) was performed to determine overall differences at each time-point for PWMT and PWTL. One-way ANOVA followed by a least-significant differences post-hoc test was used to determine differences in the mRNA levels of nNOS and iNOS among all experimental groups. All analyses were performed using SPSS 13.0 software (SPSS, Inc., Chicago, IL, USA) and $\mathrm{P}<0.05$ was considered to indicate a statistically significant difference.

\section{Results}

Pain-associated behavior in tumor-bearing mice. The PWMT and PWTL of mice in the tumor group prior to the operation were not significantly different when compared with those of the mice in the sham group. The right hind limb of the mice in the tumor group displayed a significant decrease in the PWMT at post-operative day $3(\mathrm{P}<0.05)$, and this was also observed in the mice in the sham group. At post-operative day 5, the PWMT of the two groups reclined to the basal level (Fig. 1A). Subsequently, the PWMT of mice in the tumor group showed a further decline again at day 7 , which continuously decreased until the end of the experiment on post-operative day 14 $(0.48 \pm 0.25 \mathrm{~g})$.

The mice in the tumor group showed a marked decrease in PWTL at post-operative day 3, which recovered to basal levels at days 5 and 7. Until day 7, similar trends were observed in the sham group mice. However, the PWMT of mice in the tumor group decreased again at post-operative day 10 and further declined to $10.5 \pm 3.2 \mathrm{sec}$ at day 14 , while that in the sham group remained constant (Fig. 1B). Of note, significant decreases in the PWTL at post-operative days 7-10 and in the PWMT at days 10 and 14 were observed in the tumor group when compared with those in the sham group or the time-point prior to surgery, indicating the development of marked bone cancer-associated pain.

$n N O S$ and $i N O S$ mRNA levels are increased in the spinal cords of tumor-bearing mice. The mRNA levels of nNOS were significantly increased in the spinal cord of mice in the tumor group at post-operative day 10 when compared with those in the same mice at day $7(\mathrm{P}<0.05$; Fig. $2 \mathrm{~A})$ as well as compared with 
A
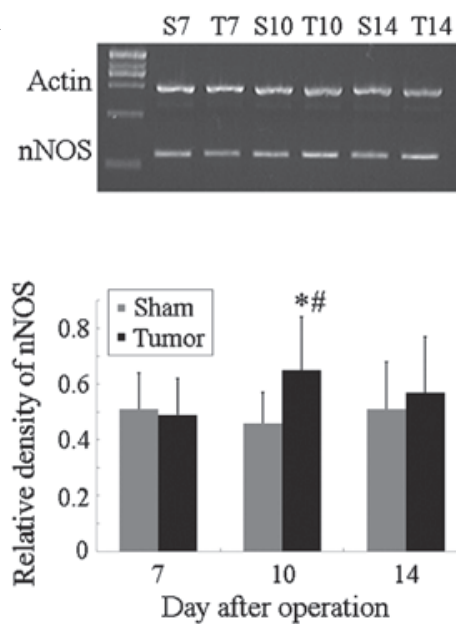

B
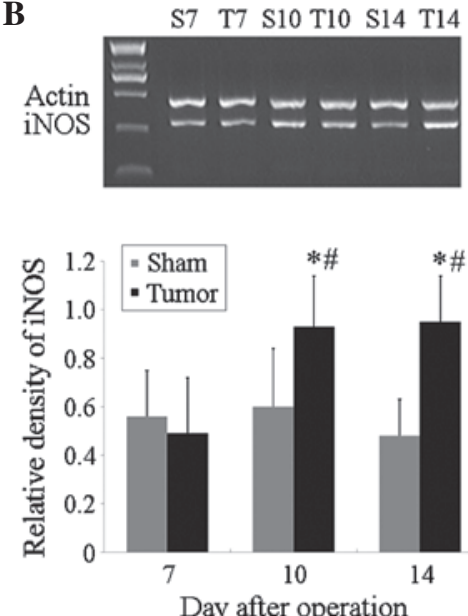

Figure 2. mRNA levels of (A) nNOS and (B) iNOS determined by reverse-transcription quantitative polymerase chain reaction with $\beta$-actin used as an internal standard. The mRNA levels of nNOS and iNOS were significantly increased at post-operative day 10 when compared with those of the controls. The increase of iNOS continued until post-operative day 14 , while that of nNOS did not. Values are expressed as the mean \pm standard deviation. ${ }^{*} \mathrm{P}<0.05$ vs. sham group at the same time-point, ${ }^{\sharp} \mathrm{P}<0.05$ vs. the same group of mice at day 7. iNOS, inducible nitric oxide synthase; nNOS, neuronal NOS; S, sham; T, tumor.

A

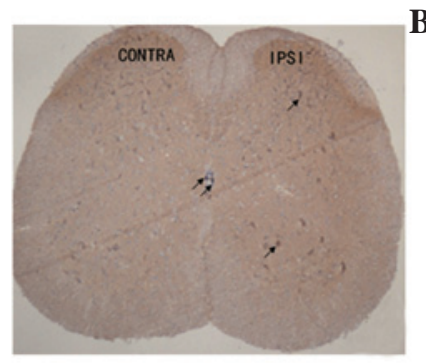

C

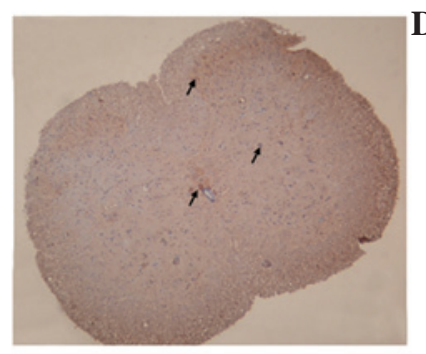

$\mathbf{E}$

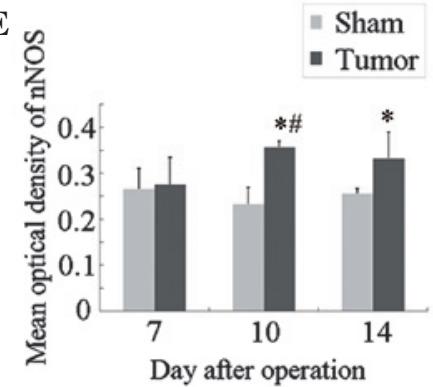

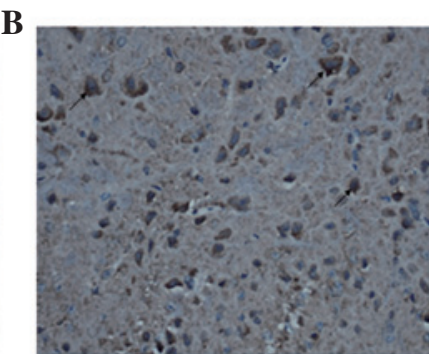
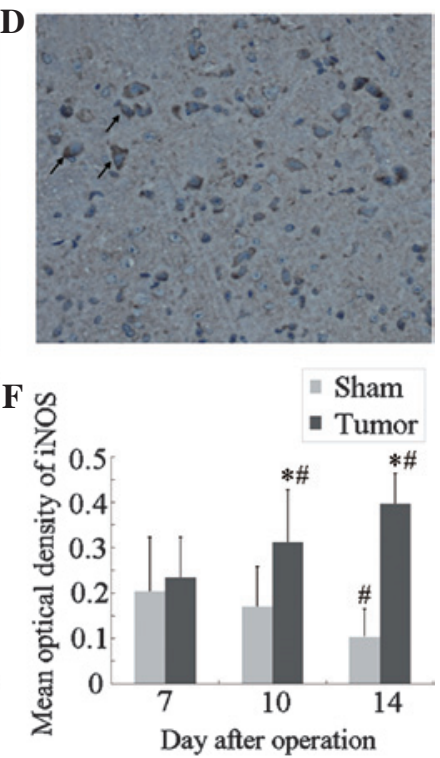

Figure 3. Immunocytochemical localization and expression of nNOS and iNOS in the spinal cord of tumor-bearing mice. (A) nNOS immunostaining in the lumbar spinal cord at day 10 after tumor-cell inoculation. (C) iNOS immunostaining in the lumbar spinal cord at day 14 after tumor-cell inoculation. Magnification, x40. The nNOS and iNOS immunoreactive cells (arrows) were mainly located in the dorsal horn of the spinal cord (laminae I-II) and a few in the ventral parts and around the central canal. (B) nNOS and (D) iNOS immunostaining in the IPSI side of the superficial dorsal horn at higher magnification, $x 200$. Statistical analysis of mean optical density of (E) nNOS and (F) iNOS. Values are expressed as the mean \pm standard deviation. ${ }^{*} \mathrm{P}<0.05$ vs. sham group at the same time-point, ${ }^{\#} \mathrm{P}<0.05$ vs. the same group of mice at day 7 . IPSI, ipsilateral; iNOS, inducible nitric oxide synthase; nNOS, neuronal NOS.

those in the sham group at day 7 , while no significant differences were present within the sham group at these time-points. Furthermore, the mRNA levels of iNOS were significantly increased in the spinal cord of mice in the tumor group at post-operative days 10 and 14 when compared with those in the sham group at the same time-points $(\mathrm{P}<0.05)$, or compared 


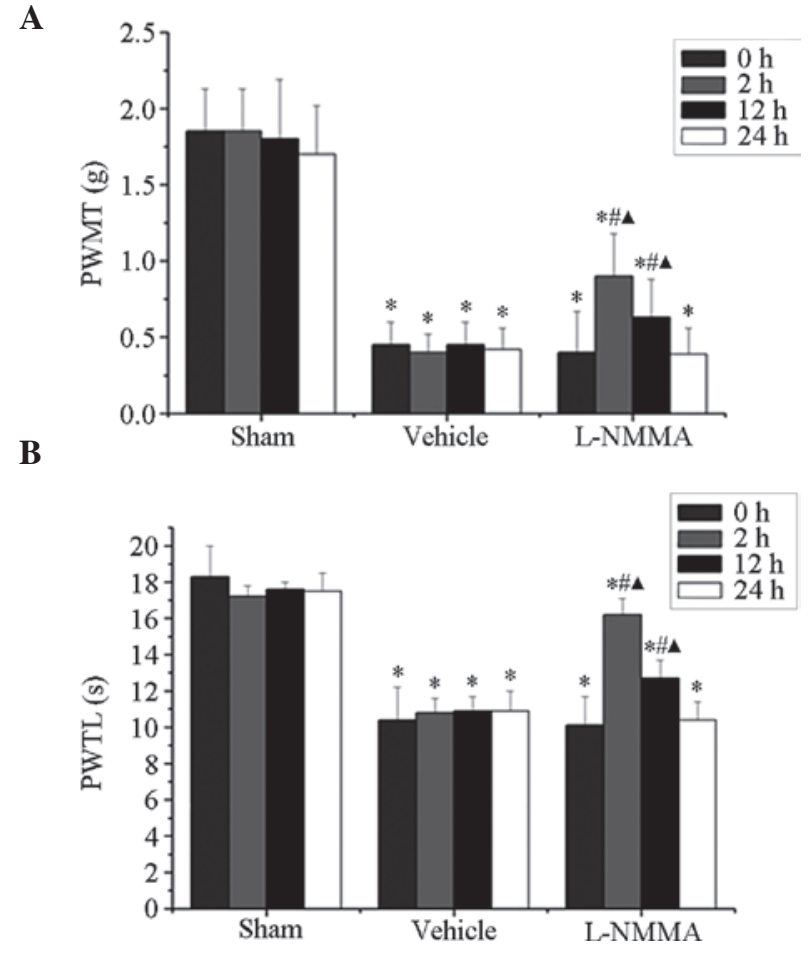

Figure 4. Effect of L-NMMA on the pain-associated behavior of mice at day 14 after tumor-cell inoculation. (A) PWMT and (B) PWTL prior to and at 2,12 and $24 \mathrm{~h}$ after intrathecal administration of $50 \mu \mathrm{g} \mathrm{L-NMMA}$ or vehicle. The mechanical and thermal hyperalgesia at post-operative day 14 induced by bone cancer was alleviated at 2 and $12 \mathrm{~h}$ after L-NMMA administration. Values are expressed as the mean \pm standard deviation. ${ }^{*} \mathrm{P}<0.05$ vs. sham group at the same time-point, ${ }^{\#} \mathrm{P}<0.05$ vs. mice treated with vehicle at the same time-point, ${ }^{\Delta} \mathrm{P}<0.05$ vs. baseline at day 0 of the same group. PWTL, paw withdrawal thermal latency; PWTM, paw withdrawal mechanical threshold.

with those in the tumor group at day 7 (Fig. 2B). However, no significant differences in the mRNA levels of iNOS were observed within the sham group at days 7-10.

Immunocytochemical localization and expression of $n N O S$ and $i N O S$ in the spinal cord of tumor-bearing mice. Next, an immunohistochemical study was performed to assess the localization and expression levels of nNOS and iNOS in the spinal cord during bone cancer development. The results showed that neurons positive for nNOS (Fig. 3A and B) and iNOS (Fig. 3C and D) were mainly located in the superficial dorsal horn (laminae I-II), which is involved in the elaboration of nociceptive stimuli, and around the central canal (lamina X) of the L3-L5 spinal cord (17). A small number of iNOS-positive neurons were observed in the ventral horn and central canal of the spinal cord.

At post-operative day 7, no significant differences in nNOS or iNOS expression were present between the sham and tumor groups (Fig. 3E and F). Compared with those in the sham group, the expression of nNOS and iNOS in the tumor group was significantly increased at post-operative day 10 and further increased at day 14. In the mice of the sham group, the expression of iNOS at day 14 was significantly decreased as compared with that at day 7 , while no significant differences in nNOS expression were observed within the sham group at different time-points.
L-NMMAalleviates bone cancerpain in mice. Atpost-operative day 14, mice in the tumor group showed a marked decline in PWMT and PWTL. Of note, this decrease was attenuated by intrathecal administration of $50 \mu \mathrm{g}$ L-NMMA (Fig. 4). The PWMT was significantly increased at $2 \mathrm{~h}(0.90 \pm 0.28$ vs. $0.40 \pm 0.27 ; \mathrm{P}<0.05)$ and $12 \mathrm{~h}(0.63 \pm 0.25$ vs. $0.40 \pm 0.27 ; \mathrm{P}<0.05)$ following administration of L-NMMA (Fig. 4A). Similarly, the PWTL was also markedly increased at $2 \mathrm{~h}(16.2 \pm 0.9$ vs. $10.1 \pm 2.3 ; \mathrm{P}<0.05)$ and $12 \mathrm{~h}(12.7 \pm 1.0$ vs. $10.1 \pm 2.3 ; \mathrm{P}<0.05)$ following administration of L-NMMA (Fig. 4B).

\section{Discussion}

The present study using a murine model of bone cancer pain following a method of a previous study by our group (16) to demonstrate that bone cancer-induced mechanical allodynia and thermal hyperalgesia are accompanied with an upregulation of nNOS and iNOS in the lumbar spinal cord. In addition, the present study found that NOS inhibitor L-NMMA alleviated cancer-induced mechanical and thermal hyperalgesia, suggesting that spinal nNOS and iNOS may participate in the development of bone cancer pain.

While the mRNA levels of nNOS and iNOS in the lumbar spinal cord of mice in the tumor group were increased at day 10 after tumor inoculation, the up-regulation of iNOS was sustained until day 14 following surgery, at which the pain-associated behavior of the mice in the tumor group were prominent and stable. The cells with immunoreactivity for nNOS and iNOS were mainly located in neurons present at the dorsal horn of the spinal cord (laminae I-II), which is an area involved in the elaboration of nociceptive stimuli, and in a few cells in the ventral parts and around the central canal (17).

It has been reported that the mRNA levels of nNOS in the lumbar spinal cord were increased at day one following surgical inflammation (25). Another study by Tang et al (5) observed a low but constitutive expression of iNOS at the mRNA and protein level in the rat spinal cord under non-stimulated conditions. They also reported that constitutively expressed spinal iNOS mediates tissue injury and inflammation-induced hyperalgesia, and that intrathecal administration of a highly selective iNOS inhibitor produced a dose-dependent inhibition of hyperalgesia. The iNOS gene is induced by bacterial lipopolysaccharide or classical pro-inflammatory cytokines, including tumor necrosis factor $\alpha$. iNOS produces a larger amount of NO over a longer time period compared with the other two constitutive enzymes, nNOS and eNOS (26). Substantial evidence has been provided for the link between the upregulation of spinal iNOS and inflammatory and neuropathic pain, suggesting a potential therapeutic value of iNOS inhibitors for such conditions $(15,27)$. In addition, Pedersen et al $(28)$ reported that an increased expression of iNOS in the dorsal horn was associated with long-term potentiation that contributed to central hyperalgesia. Consistent with a previous study (29), the results of the present study suggested that nNOS contributed to the initial NO release to facilitate nociceptive processing, while iNOS possibly participated in the persistent nociceptive responses. This observed difference in the time-course of the expression of nNOS and iNOS suggests a distinct 
contribution of NOSs to the mechanisms of bone cancer pain development.

L-NMMA, which is a non-selective inhibitor of NOS that can act on all three isoforms of NOS, has been proved to exert anti-nociceptive effects in several models of peripheral inflammation and nerve injury. Intradermal administration (30) or intrathecal injection (31) of L-NMMA has been shown to reduce, or in certain cases, to completely reverse inflammatory hyperalgesia. In addition, intrathecal injection of L-NMMA is able to reduce the mechanical allodynia evoked by nerve injury (32). Furthermore, a randomized crossover trial demonstrated the analgesic effects of L-NMMA in patients with chronic tension-type headache (33). Consistent with these studies, the present study found that the mechanical and thermal hyperalgesia at post-operative day 14 induced by bone cancer was alleviated at 2 and $12 \mathrm{~h}$ after intrathecal injection of L-NMMA, while this effect disappeared at $24 \mathrm{~h}$ after L-NMMA administration.

In conclusion, the present study demonstrated that spinal NOS may contribute to nociceptive signal processing during central sensitization in the development of bone cancer pain. As a NOS inhibitor, L-NMMA alleviated the cancer-induced mechanical allodynia and thermal hyperalgesia, suggesting its prospective application in bone cancer-associated pain. Further study is required to determine the underlying mechanism of NOS-associated bone cancer pain.

\section{Acknowledgements}

The present study was supported by the National Natural Science Foundation of China (nos. 81070892, 81171048, 81171047, 81371207, 81300950 and 81300951), the Natural Science Foundation of Jiangsu Province (no. BK2010105) and a grant from the Department of Health of Jiangsu Province of China (nos. XK201140 and RC2011006).

\section{References}

1. Colvin $\mathrm{L}$ and Fallon $\mathrm{M}$ : Challenges in cancer pain management-bone pain. Eur J Cancer 44: 1083-1090, 2008.

2. Miyamoto T, Dubin AE, Petrus MJ and Patapoutian A: TRPV1 and TRPA1 mediate peripheral nitric oxide-induced nociception in mice. PLoS One 4: e7596, 2009.

3. Naik AK, Tandan SK, Kumar D and Dudhgaonkar SP: Nitric oxide and its modulators in chronic constriction injury-induced neuropathic pain in rats. Eur J Pharmacol 530: 59-69, 2006.

4. Guan Y, Yaster M, Raja SN and Tao YX: Genetic knockout and pharmacologic inhibition of neuronal nitric oxide synthase attenuate nerve injury-induced mechanical hypersensitivity in mice. Mol Pain 3: 29, 2007.

5. Tang Q, Svensson CI, Fitzsimmons B, Webb M, Yaksh TL and Hua XY: Inhibition of spinal constitutive NOS-2 by $1400 \mathrm{~W}$ attenuates tissue injury and inflammation-induced hyperalgesia and spinal p38 activation. Eur J Neurosci 25 2964-2972, 2007.

6. Choi JI, Kim WM, Lee HG, Kim YO and Yoon MH: Role of neuronal nitric oxide synthase in the antiallodynic effects of intrathecal EGCG in a neuropathic pain rat model. Neurosci Lett 510: 53-57, 2012.

7. Lam HH, Hanley DF, Trapp BD, Saito S, Raja S, Dawson TM and Yamaguchi $\mathrm{H}$ : Induction of spinal cord neuronal nitric oxide synthase (NOS) after formalin injection in the rat hind paw. Neurosci Lett 210: 201-204, 1996.

8. Chacur M, Matos RJ, Alves AS, Rodrigues AC, Gutierrez V, Cury Y and Britto LR: Participation of neuronal nitric oxide synthase in experimental neuropathic pain induced by sciatic nerve transection. Braz J Med Biol Res 43: 367-376, 2010.
9. Lee JS, Zhang Y and Ro JY: Involvement of neuronal, inducible and endothelial nitric oxide synthases in capsaicin-induced muscle hypersensitivity. Eur J Pain 13: 924-928, 2009.

10. Handy RL and Moore PK: Effects of selective inhibitors of neuronal nitric oxide synthase on carrageenan-induced mechanical and thermal hyperalgesia. Neuropharmacology 37 : 37-43, 1998.

11. Tanabe M, Nagatani Y, Saitoh K, Takasu K and Ono H: Pharmacological assessments of nitric oxide synthase isoforms and downstream diversity of NO signaling in the maintenance of thermal and mechanical hypersensitivity after peripheral nerve injury in mice. Neuropharmacology 56: 702-708, 2009.

12. Chu YC, Guan Y, Skinner J, Raja SN, Johns RA and Tao YX: Effect of genetic knockout or pharmacologic inhibition of neuronal nitric oxide synthase on complete Freund's adjuvant-induced persistent pain. Pain 119: 113-123, 2005.

13. Toda N, Kishioka S, Hatano Y and Toda H: Modulation of opioid actions by nitric oxide signaling. Anesthesiology 110 : 166-181, 2009.

14. De Alba J, Clayton NM, Collins SD, Colthup P, Chessell I and Knowles RG: GW274150, a novel and highly selective inhibitor of the inducible isoform of nitric oxide synthase (iNOS), shows analgesic effects in rat models of inflammatory and neuropathic pain. Pain 120: 170-181, 2006

15. Sasaki A, Mabuchi T, Serizawa K, Takasaki I, Andoh T, Shiraki K, Ito S and Kuraishi Y: Different roles of nitric oxide synthase-1 and -2 between herpetic and postherpetic allodynia in mice. Neuroscience 150: 459-466, 2007.

16. Gu X, Zhang J, Ma Z, Wang J, Zhou X, Jin Y, Xia X, Gao Q and Mei F: The role of N-methyl-D-aspartate receptor subunit NR2B in spinal cord in cancer pain. Eur J Pain 14: 496-502, 2010.

17. Meller ST and Gebhart GF: Nitric oxide (NO) and nociceptive processing in the spinal cord. Pain 52: 127-136, 1993.

18. Ma ZL, Zhang W, Gu XP, Yang WS and Zeng YM: Effects of intrathecal injection of prednisolone acetate on expression of NR2B subunit and nNOS in spinal cord of rats after chronic compression of dorsal root ganglia. Ann Clin Lab Sci 37: 349-355, 2007

19. Zimmermann M: Ethical guidelines for investigations of experimental pain in conscious animals. Pain 16: 109-110, 1983.

20. Schwei MJ, Honore P, Rogers SD, Salak-Johnson JL, Finke MP, Ramnaraine ML, Clohisy DR and Mantyh PW: Neurochemical and cellular reorganization of the spinal cord in a murine model of bone cancer pain. J Neurosci 19: 10886-10897, 1999.

21. Hylden JL and Wilcox GL: Intrathecal morphine in mice: A new technique. Eur J Pharmacol 67: 313-316, 1980.

22. Chaplan SR, Bach FW, Pogrel JW, Chung JM and Yaksh TL: Quantitative assessment of tactile allodynia in the rat paw. J Neurosci Methods 53: 55-63, 1994.

23. Hargreaves K, Dubner R, Brown F, Flores C and Joris J: A new and sensitive method for measuring thermal nociception in cutaneous hyperalgesia. Pain 32: 77-88, 1988.

24. Livak KJ and Schmittgen TD: Analysis of relative gene expression data using real-time quantitative PCR and the 2(-Delta Delta C(T)) Method. Methods 25: 402-408, 2001.

25. Dolan S, Kelly JG, Huan M and Nolan AM: Transient up-regulation of spinal cyclooxygenase-2 and neuronal nitric oxide synthase following surgical inflammation. Anesthesiology 98 : 170-180, 2003.

26. Conti A, Miscusi M, Cardali S, Germanò A, Suzuki H, Cuzzocrea $S$ and Tomasello F: Nitric oxide in the injured spinal cord: Synthases cross-talk, oxidative stress and inflammation. Brain Res Rev 54: 205-218, 2007.

27. Martucci C, Trovato AE, Costa B, Borsani E, Franchi S, Magnaghi V, Panerai AE, Rodella LF, Valsecchi AE, Sacerdote $\mathrm{P}$ and Colleoni $\mathrm{M}$ : The purinergic antagonist PPADS reduces pain related behaviours and interleukin-1 beta, interleukin-6, iNOS and nNOS overproduction in central and peripheral nervous system after peripheral neuropathy in mice. Pain 137: 81-95, 2008.

28. Pedersen LM, Jacobsen LM, Mollerup S and Gjerstad J: Spinal cord long-term potentiation (LTP) is associated with increased dorsal horn gene expression of IL-1beta, GDNF and iNOS. Eur J Pain 14: 255-260, 2010.

29. Guhring H, Tegeder I, Lötsch J, Pahl A, Werner U, Reeh PW, Rehse K, Brune K and Geisslinger G: Role of nitric oxide in zymosan induced paw inflammation and thermal hyperalgesia. Inflamm Res 50: 83-88, 2001. 
30. Aley KO, McCarter G and Levine JD: Nitric oxide signaling in pain and nociceptor sensitization in the rat. J Neurosci 18 : 7008-7014, 1998.

31. Malmberg AB and Yaksh TL: Spinal nitric oxide synthesis inhibition blocks NMDA-induced thermal hyperalgesia and produces antinociception in the formalin test in rats. Pain 54: 291-300, 1993.
32. Yonehara N, Kudo C and Kamisaki Y: Involvement of NMDA-nitric oxide pathways in the development of tactile hypersensitivity evoked by the loose-ligation of inferior alveolar nerves in rats. Brain Res 963: 232-243, 2003.

33. Ashina M, Lassen LH, Bendtsen L, Jensen R and Olesen J: Effect of inhibition of nitric oxide synthase on chronic tension-type headache: A randomised crossover trial. Lancet 353: 287-289, 1999. 